\title{
APPLICATIONS OF CUTOFF RESOLVENT ESTIMATES TO THE WAVE EQUATION
}

\author{
HANS Christianson
}

\begin{abstract}
We consider solutions to the linear wave equation on non-compact Riemannian manifolds without boundary when the geodesic flow admits a filamentary hyperbolic trapped set. We obtain a polynomial rate of local energy decay with exponent depending only on the dimension.
\end{abstract}

\section{Introduction}

In this paper we study local energy decay for solutions to the linear wave equation on the non-compact Riemannian manifolds with trapping studied by NonnenmacherZworski [12]. Let $(X, g)$ be a Riemannian manifold of odd dimension $n \geq 3$ without boundary, with (non-negative) Laplace-Beltrami operator $-\Delta$ acting on functions. The Laplace-Beltrami operator is an unbounded, essentially self-adjoint operator on $L^{2}(X)$ with domain $H^{2}(X)$. We assume $(X, g)$ is asymptotically Euclidean in the sense of $[12, \S 3.2]$. That is, for $R_{0}>0$ sufficiently large, each infinite branch of $M \backslash B\left(0, R_{0}\right)$ agrees with $\mathbb{R}^{n}$, and on each branch, the semiclassical Laplacian $-h^{2} \Delta$ takes the form

$$
-\left.h^{2} \Delta\right|_{M \backslash B\left(0, R_{0}\right)}=\sum_{|\alpha| \leq 2} a_{\alpha}(x, h)\left(h D_{x}\right)^{\alpha},
$$

with $a_{\alpha}(x, h) \in \mathcal{C}_{b}^{\infty}\left(\mathbb{R}^{n}\right)$ and independent of $h$ for $|\alpha|=2$,

$$
\begin{aligned}
& \sum_{|\alpha|=2} a_{\alpha}(x, h) \xi^{\alpha} \geq C^{-1}|\xi|^{2}, \quad 0<C<\infty, \text { and } \\
& \sum_{|\alpha| \leq 2} a_{\alpha}(x, h) \xi^{\alpha} \rightarrow|\xi|^{2}, \text { as }|x| \rightarrow \infty \text { uniformly in } h .
\end{aligned}
$$

In order to quote the results of [12] we also need the following analyticity assumption: $\exists \epsilon>0$ such that the $a_{\alpha}(x, h)$ extend holomorphically to

$$
\left\{r \omega: \omega \in \mathbb{C}^{n}, \operatorname{dist}\left(\omega, \mathbb{S}^{n}\right)<\epsilon, r \in \mathbb{C},|r| \geq R_{0}, \arg r \in(-\epsilon, \epsilon)\right\},
$$

and satisfy the same estimates in this extended region. As in [12], the analyticity assumption immediately implies

$$
\partial_{x}^{\beta}\left(\sum_{|\alpha| \leq 2} a_{\alpha}(x, h) \xi^{\alpha}-|\xi|^{2}\right)=o\left(|x|^{-|\beta|}\right)\langle\xi\rangle^{2},|x| \rightarrow \infty .
$$

We assume also that the classical resolvent $\left(-\Delta-\lambda^{2}\right)^{-1}$ has a holomorphic continuation to a neighbourhood of $\lambda \in \mathbb{R}$ as a bounded operator $L_{\text {comp }}^{2} \rightarrow L_{\text {loc }}^{2}$.

Received by the editors September 4, 2007. Revision received March 31, 2009. 
We consider solutions $u$ to the following wave equation on $X \times \mathbb{R}_{t}$,

$$
\left\{\begin{array}{l}
\left(-D_{t}^{2}-\Delta\right) u(x, t)=0, \quad(x, t) \in X \times[0, \infty) \\
u(x, 0)=u_{0} \in H^{1}(X) \cap \mathcal{C}_{c}^{\infty}(X) \\
D_{t} u(x, 0)=u_{1} \in L^{2}(X) \cap \mathcal{C}_{c}^{\infty}(X)
\end{array}\right.
$$

where here $D_{t}=-i \partial_{t}$ as usual.

For $u$ satisfying (1.1) and $\chi \in \mathcal{C}_{c}^{\infty}(X)$, we define the local energy, $E_{\chi}(t)$, to be

$$
E_{\chi}(t)=\frac{1}{2}\left(\left\|\chi \partial_{t} u\right\|_{L^{2}(X)}^{2}+\|\chi u\|_{H^{1}(X)}^{2}\right) .
$$

Observe if $X=\mathbb{R}^{3}$, then the Sharp Huygens Principle tells us there exists $T$ depending only on the supports of $\chi, u_{0}$, and $u_{1}$ such that $E_{\chi}(t) \equiv 0$ for all $t \geq T$. In more general geometric settings, we study decay rates for the local energy as an analogue of the Sharp Huygens Principle. The geometry of the underlying manifold can put up serious road blocks to proving energy decay, especially if the geodesic flow has trapped sets. A classically trapped set consists of geodesics which don't escape to infinity as their arclength parameter goes to infinity. Since on a complete Riemannian manifold, geodesics are determined by an initial point and velocity, in this context we think of geodesics as curves living in phase space, or the cotangent bundle $T^{*} X$, which project onto $X$ as the usual geodesics.

Local energy for solutions to the wave equation has been well studied in various settings. Proving decay rates for local energy quantities is essential in the study of nonlinear wave equations, as well as wave-type equations in various black hole backgrounds. Morawetz [10], Lax-Morawetz-Phillips [8], and Morawetz-Ralston-Strauss [11] study the wave equation in non-trapping exterior domains in $\mathbb{R}^{n}$, showing the local energy decays exponentially in odd dimensions $n \geq 3$, and polynomially in even dimensions. This has been generalized to cases with non-trapping potentials [15] and compact non-trapping perturbations of Euclidean space [16]. In the case of elliptic trapped rays (see [14]), it is known that exponential decay of the local energy is in general not possible. Ikawa $[6,7]$ shows in dimension 3 there is exponential local energy decay with a loss in derivatives in the presence of trapped rays between convex obstacles, provided the obstacles are sufficiently small and far apart. In the case $X$ is Euclidean outside a compact set, $\partial X \neq \emptyset$, and with no assumptions on trapping, Burq shows in [1] that $E_{\chi}(t)$ decays at least logarithmically with some loss in derivatives. The author shows in [4] that if there is one hyperbolic trapped orbit with no other trapping, then the local energy decays sub-exponentially with a loss in derivative (including the case $\partial X=\emptyset$ ).

The main result of this paper is that if there is a hyperbolic trapped set which is sufficiently "thin", then the local energy decays at least polynomially, with an exponent depending on the dimension $n$. For a precise definition of trapped set, see, for example, $[12,1.5-1.6]$. The assumption that the trapped set is "thin" is quantified in the assumption in Theorem 1 that the topological pressure $P_{E}(1 / 2)<0$ (see $[12$, $3.19, \S 5.2]$ for the definition of topoligical pressure).

Theorem 1. Suppose $(X, g)$ satisfies the assumptions of the introduction, $\operatorname{dim} X=$ $n \geq 3$ is odd, and $(X, g)$ admits a compact hyperbolic fractal trapped set, $K_{E}$, in the energy level $E>0$ with topological pressure $P_{E}(1 / 2)<0$. Assume there is no other trapping and $\left(-\Delta-\lambda^{2}\right)^{-1}$ admits a holomorphic continuation to a neighbourhood 


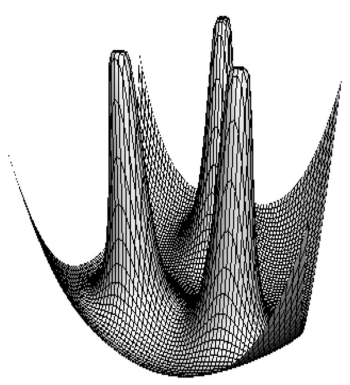

FiguRe 1. A potential $V(x)$ with three bumps.

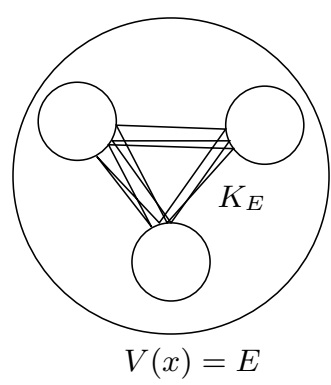

Figure 2. The level set $V(x)=E$ and a closed hyperbolic orbit in the set $K_{E}$ reflecting off the "soft" boundary.

around $\mathbb{R} \subset \mathbb{C}$. Then for each $\epsilon>0$ and $s>0$, there is a constant $C>0$, depending on $\epsilon, s$, and the supports of $\chi, u_{0}$, and $u_{1}$, such that

$$
E_{\chi}(t) \leq C\left(\frac{\log (2+t)}{\langle t\rangle}\right)^{\frac{2 s}{3 n+\epsilon}}\left(\left\|u_{0}\right\|_{H^{1+s}(X)}^{2}+\left\|u_{1}\right\|_{H^{s}(X)}^{2}\right) .
$$

Remark 1.1. At the time of publication, the author was informed by NonnenmacherZworski [13] of an improvement to the resolvent estimate in Theorem 2 to a small fixed strip in the imaginary direction, at the expense of polynomial growth in the estimate. Using Theorem 3 and Remark 3.1 one can then improve Theorem 1 to exponential decay in time. That is, under the assumptions of Theorem 1, we actually have the estimate

$$
E_{\chi}(t) \leq C_{s} e^{-t / C_{s}}\left(\left\|u_{0}\right\|_{H^{1+s}(X)}^{2}+\left\|u_{1}\right\|_{H^{s}(X)}^{2}\right) .
$$

As in [4], there are applications to the nonlinear wave equation, although we will not record them here.

As an example of where such a trapped set might occur, suppose $X$ is equal to $\mathbb{R}^{n}$ outside a compact set and $-\Delta$ is separable in at least one variable. After a conjugation, $T \Delta T^{-1}$, and a rescaling, $\lambda=1 / h$, we are left with a semiclassical operator

$$
P(h)=h D_{i} a^{i j} h D_{j}+V(x),
$$

where $V$ is a smooth, compactly supported potential. There are many potentials which admit a hyperbolic trapped set. For example, the "three-bump" potential of Figures 1 and 2 has a fractal trapped set in which each orbit is described by a finite sequence of "reflections" off the soft boundaries. That means the trapped set can be described via so-called symbolic dynamics, and hence is a Cantor-type fractal set. If the bumps are far enough apart, the set is very thin, and Theorem 1 applies.

The proof of Theorem 1 is a consequence of an adaptation of [1, Théorème 1] to this setting and the following resolvent estimates. 
Theorem 2. Suppose $(X, g)$ satisfies the assumptions of Theorem 1. Then for any $\chi \in \mathcal{C}_{c}^{\infty}(X)$ and any $\epsilon>0$ there is a constant $C=C_{\chi, \epsilon}>0$ such that

$$
\left\|\chi\left(-\Delta-\lambda^{2}\right)^{-1} \chi\right\|_{L^{2}(X) \rightarrow L^{2}(X)} \leq C \frac{\log (1+\langle\lambda\rangle)}{\langle\lambda\rangle},
$$

for

$$
\lambda \in\{\lambda:|\operatorname{Im} \lambda| \leq \Gamma(\operatorname{Re} \lambda)\} .
$$

Here $\Gamma$ is a smooth curve satisfying

$$
\Gamma(s)= \begin{cases}C_{1}, & |s| \leq C_{0}, \\ C_{2}|s|^{-3 n / 2-\epsilon}, & |s| \geq C_{0}+1,\end{cases}
$$

and $\pm \Gamma^{\prime}(s) \leq 0$ for $\pm s \geq C_{0}$, and for some choice of $C_{0}, C_{1}, C_{2}>0$.

Remark 1.2. The proof of Theorem 1 depends more on the neighbourhood in which the resolvent estimates hold than on the estimates themselves. Given a complex neghbourhood of the real axis, any polynomial cutoff resolvent estimate will give the same local energy decay rate. Theorem 2 represents a gain over the estimates in [12, Theorem 5] in the sense that the estimate holds in a complex neighbourhood of $\mathbb{R}$, rather than just on $\mathbb{R}$.

\section{Proof of Theorem 2}

To prove Theorem 2, we use the results of Nonnenmacher-Zworski [12] to prove a high energy estimate for the resolvent with complex absorbing potential, then use the holomorphic continuation to bound the cutoff resolvent by a constant for low energies. If we consider the problem

$$
\left(-\Delta-\lambda^{2}\right) u=f
$$

and restrict our attention to values $|\lambda| \geq C$ for some constant $C>0$, we can transform equation (2.1) into a semiclassical problem for fixed energy by setting

$$
\lambda=\sqrt{z} / h
$$

for $z \sim 1$ and $0<h \leq h_{0}$. Then (2.1) becomes

$$
(P-z) u=h^{2} f
$$

where

$$
P=-h^{2} \Delta
$$

is the semiclassical Laplacian.

The following Proposition is the high energy resolvent estimate from [12] with the improvement that the estimate holds in a larger neighbourhood of $\mathbb{R} \subset \mathbb{C}$.

Proposition 2.1. Suppose $W \in \mathcal{C}^{\infty}(X ;[0,1]), W \geq 0$ satisfies

$$
\operatorname{supp} W \subset X \backslash B\left(0, R_{1}\right), \quad W \equiv 1 \text { on } X \backslash B\left(0, R_{2}\right),
$$

for $R_{2}>R_{1}$ sufficiently large, and

$$
\left\|(P-i W-z)^{-1}\right\|_{L^{2} \rightarrow L^{2}} \leq C_{N}\left(1+\log (1 / h)+\frac{h^{N}}{\operatorname{Im} z}\right),
$$


for $z \in[E-\delta, E+\delta]+i(-c h, c h)$. Then for each $\epsilon>0$ and each $\chi \in \mathcal{C}_{c}^{\infty}(X)$, there is a constant $C=C_{\epsilon, \chi}>0$ such that

$$
\left\|\chi(P-z)^{-1} \chi\right\|_{L^{2} \rightarrow L^{2}} \leq C \frac{\log (1 / h)}{h}
$$

for $z \in\left[E-c_{1} h, E+c_{1} h\right]+i\left(-c_{2} h^{3 n / 2+1+\epsilon}, c_{2} h^{3 n / 2+1+\epsilon}\right)$.

We first improve [12, Lemma 9.2] in order to get cutoff resolvent estimates with the absorbing potential in a polynomial neighbourhood of the real axis. The proof of the following lemma is an adaptation of the "three-lines" theorem from complex analysis and borrows techniques from $[3,2,12]$ and the references cited therein.

Lemma 2.2. Suppose $F(z)$ is holomorphic in a neighbourhood of

$$
\Omega=[-1,1]+i\left(-c_{-}, c_{+}\right),
$$

and satisfies

$$
\begin{aligned}
\log |F(z)| & \leq M, z \in \Omega \\
|F(z)| & \leq \alpha+\frac{\gamma}{\operatorname{Im} z}, z \in \Omega \cap\{\operatorname{Im} z>0\} .
\end{aligned}
$$

Then if $\gamma \leq \epsilon M^{-3 / 2}$ for $\epsilon>0$ sufficiently small, there exists a constant $C=C_{\epsilon}>0$ such that

$$
|F(z)| \leq C \alpha, \quad z \in[-1 / 2,1 / 2]+i\left(-M^{-3 / 2}, M^{-3 / 2}\right) .
$$

Proof. Choose $\psi(x) \in \mathcal{C}_{c}^{\infty}([-1,1]), \psi \equiv 1$ on $[-1 / 2,1 / 2]$, and set

$$
\varphi(z)=\beta^{-1 / 2} \int e^{-(x-z-i c \beta)^{2} / \beta} \psi(x) d x,
$$

where $0<\beta<1$ and $c>0$ will be chosen later. The function $\varphi(z)$ enjoys the following properties:

(a) $\varphi(z)$ is holomorphic in $\Omega$,

(b) $|\varphi(z)| \leq C$ on $\Omega \cap\left\{|\operatorname{Im} z| \leq \beta^{1 / 2}\right\}$,

(c) $|\varphi(z)| \geq C^{-1}$ on $\{|\operatorname{Re} z| \leq 1 / 2\} \cap\{|\operatorname{Im} z| \leq \beta\}$ if $c>0$ is chosen appropriately,

(d) $|\varphi(z)| \leq C e^{-C / \beta}$ for $z \in\{ \pm 1\}+i\left(-\beta^{1 / 2}, \beta^{1 / 2}\right)$.

Now for $a \in \mathbb{R}$ to be determined, set

$$
g(z)=e^{i a z} \varphi(z) F(z) .
$$

For $\delta_{ \pm}>0$ to be determined, let

$$
\Omega^{\prime}:=\Omega \cap\left\{-\delta_{-} \leq \operatorname{Im} z \leq \delta_{+}\right\} .
$$

We have the following bounds for $g(z)$ on the boundary of $\Omega^{\prime}$ :

$$
\log |g(z)| \leq \begin{cases}-C / \beta+M-a \operatorname{Im} z, & \operatorname{Re} z= \pm 1, \text { if }|\operatorname{Im} z| \leq \beta^{1 / 2} \\ C+M+a \delta_{-}, & \operatorname{Im} z=-\delta_{-} \geq-\beta^{1 / 2} \\ C+\log \left(\alpha+\gamma / \delta_{+}\right)-a \delta_{+}, & \operatorname{Im} z=\delta_{+} \leq \beta^{1 / 2}\end{cases}
$$

We want to choose $a, \beta$, and $\delta_{ \pm}$to optimize these inequalities. Choosing $a=-2 M / \delta_{-}$ yields

$$
\log |g(z)| \leq C-M \text { for } \operatorname{Im} z=-\delta_{-},
$$


and choosing $\delta_{+}=|2 / a|$ yields

$$
\log |g(z)| \leq C+\log \left(\alpha+\gamma / \delta_{+}\right)+2, \text { for } \operatorname{Im} z=\delta_{+} .
$$

Finally, chooing $\beta=C^{\prime} / M$ for an appropriate $C^{\prime}>0$ yields

$$
\log |g(z)| \leq-C^{-1} M \text { for } \operatorname{Re} z= \pm 1,|\operatorname{Im} z| \leq \max \left\{\delta_{+}, \delta_{-}\right\},
$$

and taking $\delta_{-}=C^{\prime \prime} M^{-1 / 2}, \delta_{+}=C^{\prime \prime} M^{-3 / 2}$ gives

$$
\log |g(z)| \leq C^{\prime \prime \prime}+\log \left(\alpha+\gamma / \delta_{+}\right) \text {on } \partial \Omega^{\prime} .
$$

In order to conclude the stated inequality on $F(z)$, we need to invert $e^{-i a z} \varphi(z)$, which, from the definition of $a$ and the properties of $\varphi$ stated above, is possible for

$$
z \in[-1 / 2,1 / 2]+i\left(-M^{-3 / 2}, M^{-3 / 2}\right) .
$$

Then for $z$ in this range and $\gamma$ satisfying $\gamma \leq \epsilon M^{-3 / 2}$,

$$
|F(z)| \leq C \alpha(1+\epsilon) \leq C^{\prime} \alpha,
$$

as claimed.

Now to prove Proposition 2.1, as in [12], we apply Lemma 2.2 to

$$
F(\zeta)=\left\langle h(P-i W-h \zeta)^{-1} f, g\right\rangle_{L^{2}},
$$

for $f, g \in L^{2}$. For $M$ we use the well-known estimate (see, for example [5, Lemma $6.1])$

$$
\left\|(P-i W-z)^{-1}\right\|_{L^{2} \rightarrow L^{2}} \leq C_{\epsilon} e^{C h^{-n-\epsilon}}, \quad \operatorname{Im} z \geq-h / C,
$$

and take $M=C_{\epsilon} h^{-n-\epsilon}$. For the other parameters, we take

$$
\gamma=h^{N}, \quad \alpha=c_{0}+\log (1 / h) .
$$

Rescaling, we conclude

$$
\left\|(P-i W-z)^{-1}\right\| \leq C \frac{\log (1 / h)}{h}
$$

in the stated region. Then we apply the remainder of the proof [12, Theorem 5].

\section{Proof of Theorem 1}

In this section we adapt the proof of [1, Théorème 1] to the case where one has better resolvent estimates. Theorem 1 follows from a more abstract theorem on semigroups (see [1, Théorème 3] and [9]). In order to motivate this theorem, let us see what is needed for the proof of Theorem 1.

Set

$$
B=\left(\begin{array}{cc}
0 & -i \text { id } \\
-i \Delta & 0
\end{array}\right)
$$

so that if

$$
U_{0}:=\left(u_{0}, u_{1}\right) \in H:=H^{1}(X) \times L^{2}(X)
$$

is smooth with compact support, and $u$ solves (1.1),

$$
U(t):=\left(\begin{array}{c}
u(t) \\
\partial_{t} u(t)
\end{array}\right)=e^{i t B} U_{0} .
$$


We note also that for $s \in \mathbb{R}$ we have

$$
\left\|u_{0}\right\|_{H^{s+1}}+\left\|u_{1}\right\|_{H^{s}} \simeq\left\|(1-i B)^{s} U_{0}\right\|_{H},
$$

so that if $\chi_{1}, \chi_{2} \in \mathcal{C}_{c}^{\infty}(X)$ and $\chi_{2} \equiv 1$ on $\operatorname{supp} U_{0}$, to prove Theorem 1 , we want to estimate

$$
\left\|\chi_{1} e^{i t B} U_{0}\right\|_{H^{s+1} \times H^{s}}=\left\|\chi_{1} e^{i t B} \chi_{2} U_{0}\right\|_{H^{s+1} \times H^{s}} .
$$

We observe the commutator $\left[\chi_{2}, B\right]$ is compactly supported and bounded on $H$, so that we are left to estimate

$$
\begin{aligned}
\left\|\chi_{1} e^{i t B} \chi_{2}\right\|_{H^{s+1} \times H^{s} \rightarrow H} & =\left\|\chi_{1} e^{i t B} \chi_{2}(1-i B)^{-s}\right\|_{H \rightarrow H} \\
& \leq C\left\|\chi_{1} e^{i t B}(1-i B)^{-s} \tilde{\chi}_{2}\right\|_{H \rightarrow H},
\end{aligned}
$$

where $\tilde{\chi}_{2} \in \mathcal{C}_{c}^{\infty}(X)$ is supported on a slightly larger set than $\chi_{2}$. Hence if we can show

$$
\left\|\chi_{1} e^{i t B}(1-i B)^{-s} \tilde{\chi}_{2}\right\|_{H \rightarrow H} \leq C\left(\frac{\langle t\rangle}{\log (2+t)}\right)^{\frac{-2 s}{3 n+\epsilon}},
$$

we are almost done. This estimate follows from the next theorem, upon taking (in the notation below), $k=2, P(t)=t^{-3 n / 2-\epsilon / 2}$, and

$$
F(t)=\left(\frac{t}{\log t}\right)^{\frac{4}{3 n+\epsilon}}
$$

Finally, we observe the spaces $H^{1+s} \times H^{s}$ are complex interpolation spaces, hence interpolating with the trivial estimate

$$
E_{\chi}(t) \leq C\left(\left\|u_{0}\right\|_{H^{1}}^{2}+\left\|u_{1}\right\|_{L^{2}}^{2}\right)
$$

yields (1.2) for $s \geq 0$.

This motivates trying to estimate quantities involving semigroups and cutoff resolvents of the form

$$
\left\|\chi_{1} e^{i t B}(1-i B)^{-s} \chi_{2}\right\|_{H \rightarrow H},
$$

where $\chi_{j}: H \rightarrow H$ are bounded operators on a Hilbert space $H$ and the resolvent satisfies some analyticity assumptions. For this we develop the following more general theorem similar to [1, Théorème 3] and [9].

Let $H$ be a Hilbert space and let $B$ be an unbounded linear operator on $H$. Assume for $\operatorname{Im} \xi \leq 0$,

$$
\operatorname{Im}(B u, u)_{H} \geq 0 .
$$

Let $\operatorname{Dom}(B)=\operatorname{Dom}(1-i B)$ denote the domain of $B$. Assume for $\operatorname{Im} \xi<0, \xi-B$ is bijective and bounded with respect to the natural norm on $\operatorname{Dom}(B)$,

$$
\|u\|_{\text {Dom }(B)}^{2}=\|u\|_{H}^{2}+\|B u\|_{H}^{2},
$$

and

$$
\left\|(\xi-B)^{-1}\right\|_{H \rightarrow H} \leq C|\operatorname{Im} \xi|^{-1} .
$$


By the Hille-Yosida Theorem, for every $k \in \mathbb{N}$ and $s \geq 0$, we can construct the operators

$$
\frac{e^{i s B}}{(1-i B)^{k}}
$$

where $e^{i s B}$ satisfies the evolution equation

$$
\left\{\begin{array}{l}
\left(D_{s}-B\right) e^{i s B}=0 \\
\left.e^{i s B}\right|_{s=0}=\mathrm{id} .
\end{array}\right.
$$

Now suppose $\chi_{j}, j=1,2$ are bounded operators $H \rightarrow H$, and $\chi_{1}(\xi-B)^{-1} \chi_{2}$ continues holomorphically to a neighbourhood of the region

$$
\Omega=\left\{\xi \in \mathbb{C}:|\operatorname{Im} \xi| \leq\left\{\begin{array}{ll}
C_{1}, & |\operatorname{Re} \xi| \leq C_{2} \\
P(|\operatorname{Re} \xi|), & |\operatorname{Re} \xi| \geq C_{2},
\end{array}\right\},\right.
$$

where $P(|\operatorname{Re} \xi|)>0$ and is monotone decreasing (or constant) as $|\operatorname{Re} \xi| \rightarrow \infty$, $P\left(C_{2}\right)=C_{1}$, and assume for simplicity that $\partial \Omega$ is smooth. Assume

$$
\left\|\chi_{1}(\xi-B)^{-1} \chi_{2}\right\|_{H \rightarrow H} \leq G(|\operatorname{Re} \xi|)
$$

for $\xi \in \Omega$, where $G(|\operatorname{Re} \xi|)=\mathcal{O}\left(|\operatorname{Re} \xi|^{N}\right)$ for some $N \geq 0$. We further assume that the propagator $e^{i s B}$ "acts finitely locally," in the sense that for $s \in[0,1]$,

$$
\tilde{\chi}_{2}:=e^{i s B} \chi_{2} e^{-i s B}
$$

is also a bounded operator on $H$, and $\chi_{1}(\xi-B)^{-1} \tilde{\chi}_{2}$ continues holomorphically to a neighbourhood of $\Omega$ and still satisfies the estimate (3.2), possibly with $G$ replaced by $C G$ for a constant $C>0$.

Theorem 3. Suppose $B$ satisfies all the assumptions above, and let $k \in \mathbb{N}, k>N+1$. Then for any $F(t)>0$, monotone increasing, satisfying

$$
F(t)^{(k+1) / 2} \leq \exp (t P(F(t))),
$$

there is a constant $C>0$ depending on the supports of $\chi_{1}$ and $\chi_{2}$ such that

$$
\left\|\chi_{1} \frac{e^{i t B}}{(1-i B)^{k}} \chi_{2}\right\|_{H \rightarrow H} \leq C F(t)^{-k / 2} .
$$

Remark 3.1. Evidently, if we have polynomial resolvent bounds in a fixed strip around the real axis, we have exponential local energy decay for the wave equation with a loss in derivatives.

Further, Theorem 3 applies if $X$ is a compact manifold, $H=H^{1}(X) \times L^{2}(X)$, and

$$
B=\left(\begin{array}{cc}
0 & -i \mathrm{id} \\
-i \Delta_{g} & 2 i a(x)
\end{array}\right)
$$

which is the the matrix for the first order system associated to the damped wave equation. In this situation if $a$ controls $X$ geometrically except for in a neighbourhood of a single closed hyperbolic geodesic (see [3, Corollary 8]), there are indeed polynomial resolvent bounds in a fixed strip. We can take $\chi_{1}=\chi_{2}=1$ to conclude there is exponential energy decay with loss in derivatives for solutions to the damped wave equation. This corrects a mistake in the proof of [3, Theorem 5].

To put these estimates in perspective, we recall that in the original paper of Burq [1] there were no assumptions on trapping, but $\partial X \neq \emptyset$. In this case, there can 
be resonances near 0 , so the domain to which the cutoff resolvent extends is more complicated. Roughly, the cutoff resolvent extends for large $\operatorname{Re} \xi$ to a region in the upper half plane bounded by $e^{-|\operatorname{Re} \xi| / C}$, with exponentially growing bounds. There is also possibly an angular sector containing the origin in the upper half plane where the resolvent does not continue. In this paper we have much better bounds (as mentioned above, polynomial bounds are all we need), and a polynomial neighbourhood of the real axis to which the cutoff resolvent extends. This explains why the estimate in this paper is stronger, but of course under more assumptions.

Before proceeding with the proof of Theorem 3, we first need a lemma, which gives a nice representation of the propagator as a contour integral.

Lemma 3.2. For $k \geq 2$, the propagator satisfies the following identity on $H$ :

$$
\frac{e^{i t B}}{(1-i B)^{k}}=\frac{1}{2 \pi i} \int_{\operatorname{Im} \xi=-\frac{1}{2}} e^{i t \xi}(1-i \xi)^{-k}(\xi-B)^{-1} d \xi
$$

Proof. We write $I_{k}$ for the right hand side and observe both the left hand side and $I_{k}$ satisfy the evolution equation

$$
\left(D_{t}-B\right) w=0 .
$$

To calculate $I_{k}(0)$, we deform the contour to see

$$
I_{k}(0)=\frac{1}{2 \pi i}\left(\int_{\operatorname{Im} \xi=-C}-\int_{\partial D(-i, \epsilon)}\right)(1-i \xi)^{-k}(\xi-B)^{-1} d \xi .
$$

Letting $C \rightarrow \infty$, the first integral vanishes. Thus we need to calculate the second integral, which actually makes sense for $k \geq 1$. For $k=1$, this is the residue formula, while for $k>1$ the formula follows by induction and the continuity of $(\xi-B)^{-1}$ as $\epsilon \rightarrow 0$.

Thus the left hand side and $I_{k}$ have the same initial conditions, and the lemma is proved.

Proof of Theorem 3. The idea of the proof of Theorem 3 is to introduce a cutoff to times $t>0$. This has the effect that, upon differentiation in $t$, a solution to a homogeneous equation (e.g. the propagator (3.1)) becomes inhomogeneous, allowing us to use Duhamel's formula to write an integral representation of the solution. Having done this, we estimate the "high-frequency" part of this integral separately from the "low-frequency" part, and then finally choose an appropriate definition of "highfrequency" which optimizes these two estimates.

For an initial condition $u_{0} \in H$, let $V(t)=e^{i t B} \chi_{2} u_{0}$, and consider $U(t)=\psi(t)(1-$ $i B)^{-k} V(t)$ for $\psi(t) \in \mathcal{C}^{\infty}(\mathbb{R})$ satisfying $\psi \equiv 0$ for $t \leq 1 / 3, \psi \equiv 1$ for $t \geq 2 / 3$, and $\psi^{\prime} \geq 0$. We observe by the sub-unitarity of $e^{i t B}$ for $t \geq 0$,

$$
\|U(t)\| \leq C\|V(t)\| \leq C^{\prime}\left\|u_{0}\right\|,
$$

where for the remainder of the proof, $\|\cdot\|=\|\cdot\|_{H}$ unless otherwise specified.

The family $U(t)$ satisfies

$$
\left(D_{t}-B\right) U=\tilde{A}(t)(1-i B)^{-k} V(t),
$$


where $\tilde{A}$ is a bounded operator on $H$ with support contained in $[1 / 3,2 / 3]$. As $U(0)=$ 0 , Duhamel's formula yields

$$
U(t)=\int_{0}^{t} e^{i(t-s) B} \tilde{A}(s)(1-i B)^{-k} V(s) d s,
$$

and by Lemma 3.2 ,

$$
U(t)=\int_{s=0}^{t} \int_{\operatorname{Im} \xi=-1 / 2} e^{i(t-s) \xi} \tilde{A}(s)(1-i \xi)^{-k}(\xi-B)^{-1} V(s) d \xi d s .
$$

For a function $F(t)>0$, monotone increasing in $t$ to be selected later, we will cut off frequencies in $|\xi|$ above and below $F(t)$. We convolve with a Gaussian to smooth this out:

$$
\begin{aligned}
U(t) & =\int_{s=0}^{t} \int_{\operatorname{Im} \xi=-1 / 2} \int_{\lambda} e^{i(t-s) \xi} \tilde{A}(s)(1-i \xi)^{-k}(\xi-B)^{-1} \\
& =\int_{0}^{t} \int_{\operatorname{Im} \xi=-1 / 2}\left(\int_{|\lambda| \leq F^{\frac{1}{2}}(t)}+\int_{|\lambda| \geq F^{\frac{1}{2}}(t)} e^{-c_{0}\left(\lambda-\xi / F^{\frac{1}{2}}(t)\right)^{2}} V(s) d \lambda d \xi d s\right. \\
& =: \quad I_{1}+I_{2},
\end{aligned}
$$

where $c_{0}$ is a constant to be fixed later.

Claim 1: For $A>0$ to be chosen later, there exist constants $C, c_{2}>0$ such that $I_{1}$ as defined above satisfies

$$
\left\|\chi_{1} I_{1}\right\| \leq C \max \left\{\begin{array}{l}
F^{\frac{1}{2}}(t) e^{-t P(A F(t))} \\
F^{\frac{1}{2}}(t) e^{-c_{2} F(t)}
\end{array}\right\}\left\|u_{0}\right\| .
$$

Proof of Claim 1. From the resolvent and propagator continuation properties (3.2), the integrand in $\chi_{1} I_{1}$ is holomorphic in $\{-1<\operatorname{Im} \xi<0\} \cup$ neigh $\Omega$. Observe that since $\lambda^{2} \leq F(t)$, and

$$
\frac{\lambda}{F^{\frac{1}{2}}(t)} \leq 1
$$

for $|\operatorname{Re} \xi| \gg F(t)$ and $\operatorname{Im} \xi$ bounded, we have the estimate

$$
\begin{aligned}
\left(\lambda-\xi / F^{\frac{1}{2}}(t)\right)^{2} & =\lambda^{2}-\frac{2 \lambda \xi}{F^{\frac{1}{2}}(t)}+\frac{\xi^{2}}{F(t)} \\
& \sim \frac{\xi^{2}}{F(t)} \\
& \gg|\xi|,
\end{aligned}
$$

and the integrand is rapidly decaying as $|\operatorname{Re} \xi| \rightarrow \infty$ in $\mathbb{R}$. Hence we can deform the contour in $\xi$ to

$$
\Gamma=\left\{\xi \in \mathbb{C}: \operatorname{Im} \xi=\left\{\begin{array}{ll}
C_{1}, & |\operatorname{Re} \xi| \leq C_{2} \\
P(|\operatorname{Re} \xi|), & |\operatorname{Re} \xi| \geq C_{2} .
\end{array}\right\}\right.
$$


Let $A>0$ be a parameter to be fixed later. We further break $I_{1}$ into integrals where $\operatorname{Re} \xi$ is larger than or smaller than $A F(t)$ :

$$
\begin{aligned}
\chi_{1} I_{1} & =\chi_{1} \int_{0}^{t}\left(\int_{\Gamma \cap\{|\operatorname{Re} \xi| \leq A F(t)\}}+\int_{\Gamma \cap\{|\operatorname{Re} \xi| \geq A F(t)\}}\right) \int_{|\lambda| \leq F^{\frac{1}{2}}(t)}(\cdot) d \lambda d \xi d s \\
& =: \quad \chi_{1} J_{1}+\chi_{1} J_{2} .
\end{aligned}
$$

For $J_{1}$, if $t \geq 2$, since $P(|\operatorname{Re} \xi|)$ is monotone decreasing, we have

$$
\operatorname{Im} \xi \geq P(A F(t))
$$

and on the support of $\tilde{A}(s)$, we have $t-s \geq t-1$. Hence if $k>N+1$,

$$
\begin{aligned}
\left\|\chi_{1} J_{1}\right\| & \leq C \int_{\Gamma \cap\{|\operatorname{Re} \xi| \leq A F(t)\}} \int_{|\lambda| \leq F^{\frac{1}{2}}(t)} e^{-(t-1) P(A F(t))}\langle\xi\rangle^{-k} G(|\operatorname{Re} \xi|) \\
& \cdot\left|e^{-c_{0}\left(\lambda-\xi / F^{\frac{1}{2}}(t)\right)^{2}}\right| d \lambda d \xi\left\|u_{0}\right\| \\
& \leq C A F(t) e^{-t P(A F(t))}\left\|u_{0}\right\| .
\end{aligned}
$$

For $J_{2}$, we observe that, again since $|\lambda| \leq F^{\frac{1}{2}}(t)$, for $A$ large enough and $|\operatorname{Re} \xi| \geq$ $A F(t)$,

$$
\begin{aligned}
\operatorname{Re}\left(\lambda-\xi / F^{\frac{1}{2}}(t)\right)^{2} & =\lambda^{2}-\frac{2 \lambda \operatorname{Re} \xi}{F^{\frac{1}{2}}(t)}+\frac{(\operatorname{Re} \xi)^{2}-(\operatorname{Im} \xi)^{2}}{F(t)} \\
& \geq C^{-1}\left(\lambda^{2}+(\operatorname{Re} \xi)^{2} / F(t)\right)
\end{aligned}
$$

Hence,

$$
\begin{aligned}
\left\|\chi_{1} J_{2}\right\| & \leq C \int_{\Gamma \cap\{|\operatorname{Re} \xi| \geq A F(t)\}} \int_{|\lambda| \leq F^{\frac{1}{2}}(t)}\langle\xi\rangle^{-k} G(|\operatorname{Re} \xi|) \\
& \leq e^{-c_{0}\left(\lambda-\xi / F^{\frac{1}{2}}(t)\right)^{2}} \mid d \lambda d \xi\left\|u_{0}\right\| \\
& \leq C \int_{|\eta| \geq F^{\frac{1}{2}}(t)} F^{\frac{1}{2}}(t) e^{-c_{1} \eta^{2}} d \eta\left\|u_{0}\right\| \\
& C F^{\frac{1}{2}}(t) e^{-c_{2} F(t)}\left\|u_{0}\right\| .
\end{aligned}
$$

Claim 2: We now claim there exists a constant $C>0$ such that

$$
\left\|\chi_{1} I_{2}\right\| \leq C \max \left\{\begin{array}{l}
C\left(e^{-F(t)}+e^{-F^{\frac{1}{2}}(t) / 3}\right) \\
C\left(F(t)^{-k / 2}+e^{-F(t) / C}\right)
\end{array}\right\}\left\|u_{0}\right\| .
$$

Proof of Claim 2. Now since $\tilde{A}(s)$ is only supported in $[1 / 3,2 / 3]$, the integrand in the definition of $I_{2}(t)$ is independent of $s$ for $s \geq 2 / 3$. Hence if we set

$$
\begin{array}{r}
J(\tau)=\int_{s=0}^{1} \int_{\substack{\operatorname{Im} \xi=-1 / 2 \\
|\lambda| \geq F^{1 / 2}(t)}} \tilde{A}(s) e^{i(\tau-s) \xi}(1-i \xi)^{-k}(\xi-B)^{-1} \\
\cdot\left(c_{0} / \pi\right)^{\frac{1}{2}} e^{-c_{0}\left(\lambda-\xi / F^{\frac{1}{2}}(t)\right)^{2}} V(s) d \lambda d \xi d s
\end{array}
$$


we have $J(\tau) \equiv I_{2}(\tau)$ for $\tau \geq 1$. Observe

$$
\begin{aligned}
\left(D_{\tau}-B\right) J(\tau) & =\int_{s=0}^{1} \int_{\substack{\operatorname{Im} \xi=-1 / 2 \\
|\lambda| \geq F^{1 / 2}(t)}} \tilde{A}(s) e^{i(\tau-s) \xi}(1-i \xi)^{-k} \\
& =: \quad K(\tau) .
\end{aligned}
$$

Hence

$$
J(t)=e^{i t B} J(0)+\int_{0}^{t} e^{i(t-s) B} K(s) d s .
$$

Again, by the subunitarity of the propagator, we need to estimate $\left\|\chi_{1} J(0)\right\|$ and $\int_{0}^{t}\left\|\chi_{1} K(s)\right\| d s$.

For $J(0)$, we first consider $\lambda \geq F^{\frac{1}{2}}(t)$. Since $k>N+1$, we can deform the $\xi$-contour to

$$
\Gamma^{\prime}=\Gamma_{-} \cup \Gamma_{+}
$$

where

$$
\begin{aligned}
\Gamma_{-}= & \{\operatorname{Re} \xi \leq F(t) / A, \operatorname{Im} \xi=-1 / 2\} \\
& \cup\left\{\operatorname{Re} \xi=F(t) / A,-F^{\frac{1}{2}}(t) \leq \operatorname{Im} \xi \leq-1 / 2\right\}
\end{aligned}
$$

and

$$
\Gamma_{+}=\left\{\operatorname{Re} \xi \geq F(t) / A, \operatorname{Im} \xi=-F^{\frac{1}{2}}(t)\right\} .
$$

If $\xi \in \Gamma_{-}$, we have

$$
\operatorname{Re}\left(\lambda-\xi / F^{\frac{1}{2}}(t)\right)^{2} \geq \lambda^{2} / C,
$$

so

$$
\int_{\xi \in \Gamma_{-}} \int_{\lambda \geq F^{\frac{1}{2}}(t)}\langle\xi\rangle^{-k} G(|\operatorname{Re} \xi|) \cdot e^{-c_{0}\left(\lambda-\xi / F^{\frac{1}{2}}(t)\right)^{2}} V(s) d \lambda d \xi \leq C e^{-F(t)}
$$

For $\xi \in \Gamma_{+}$, we have

$$
\left|e^{-i s \xi}\right|=e^{-F^{\frac{1}{2}}(t) / 3},
$$

and since the remaining integrand in $\left\|\chi_{1} J(0)\right\|$ is integrable, the contribution to $\left\|\chi_{1} J(0)\right\|$ coming from $\lambda \geq F^{\frac{1}{2}}(t)$ is bounded by

$$
C\left(e^{-F(t)}+e^{-F^{\frac{1}{2}}(t) / 3}\right)\left\|u_{0}\right\| .
$$

The contribution to $\left\|\chi_{1} J(0)\right\|$ coming from $\lambda \leq-F^{\frac{1}{2}}(t)$ is handled similarly to obtain the same bound.

For $s \in[1, t]$, since $k>N+1$, we can deform the $\xi$-contour in the definition of $K$ to $\operatorname{Im} \xi=F^{\frac{1}{2}}(t)$. Then for this range of $s$,

$$
\left\|\chi_{1} K(s)\right\| \leq C \int_{\eta} e^{-(s-2 / 3) F^{\frac{1}{2}}(t)}\langle\eta\rangle^{-k} d \eta\left\|u_{0}\right\|,
$$

and hence

$$
\int_{1}^{t}\left\|\chi_{1} K(s)\right\| d s \leq C F^{\frac{1}{2}}(t)^{-1} e^{-F^{\frac{1}{2}}(t) / 3}
$$



write

We have yet to estimate $\int_{0}^{1}\left\|\chi_{1} K(s)\right\| d s$. For this we use Plancherel's formula to

$$
\begin{aligned}
& \left(\int_{0}^{1}\left\|\chi_{1} K(s)\right\| d s\right)^{2} \\
& \leq \int_{-\infty}^{\infty}\left\|\chi_{1} K(s)\right\|^{2} d s \\
& \quad=\int_{-\infty}^{\infty}\left\|(1-i \xi)^{-k} \widehat{\tilde{A} V}(\xi) \int_{|\lambda| \geq F^{\frac{1}{2}}(t)} e^{-c_{0}\left(\lambda-\xi / F^{\frac{1}{2}}(t)\right)^{2}} d \lambda\right\|^{2} d \xi
\end{aligned}
$$

If we estimate this integral by again considering regions where $|\xi| \leq F(t) / A$ and $|\xi| \geq F(t) / A$ respectively, we see (3.5) is majorized by

$$
\begin{aligned}
& C\left(e^{-F(t) / C}+F(t)^{-k}\right) \int_{-\infty}^{\infty}\|\widehat{\tilde{A} V}(\xi)\|^{2} d \xi \\
& =C\left(e^{-F(t) / C}+F(t)^{-k}\right) \int_{-\infty}^{\infty}\|\tilde{A} V(s)\|^{2} d s \\
& \leq C\left(e^{-F(t) / C}+F(t)^{-k}\right)\left\|u_{0}\right\|^{2} .
\end{aligned}
$$

To finish the proof of Theorem 3, we combine all of the above estimates to get

$$
\left\|\chi_{1} U(t)\right\| \leq C \max \left\{\begin{array}{l}
F(t)^{-k / 2} \\
e^{-F^{\frac{1}{2}}(t) / 3}+e^{-F(t) / C}, \\
F^{\frac{1}{2}}(t) e^{-F^{\frac{1}{2}}(t) / 3}, \\
F^{\frac{1}{2}}(t) e^{-t P(A F(t))}+F^{\frac{1}{2}}(t) e^{-F^{\frac{1}{2}}(t)}
\end{array}\right\}\left\|u_{0}\right\| .
$$

Optimizing these estimates with respect to $F$ recovers (3.3), which then yields (3.4).

\section{Acknowledgements}

This research was partially conducted during the period the author was employed by the Clay Mathematics Institute as a Liftoff Fellow. The author would like to thank the anonymous referee whose comments and suggestions have helped improve the exposition.

\section{References}

[1] N. Burq, Décroissance de l'énergie locale de l'équation des ondes pour le problème extérieur et absence de résonance au voisinage du réel, Acta Math. 180 (1998), no. 1, 1-29.

[2] N. Burq and M. Zworski, Geometric control in the presence of a black box, J. Amer. Math. Soc. 17 (2004), no. 2, 443-471 (electronic).

[3] H. Christianson, Semiclassical non-concentration near hyperbolic orbits, J. Funct. Anal. 246 (2007), no. 2, 145-195.

[4] - Dispersive Estimates for Manifolds with One Trapped Orbit, Comm. Partial Differential Equations 33 (2008) 1147-1174.

[5] N. Dencker, J. Sjöstrand, and M. Zworski, Pseudospectra of semiclassical (pseudo-) differential operators, Comm. Pure Appl. Math. 57 (2004), no. 3, 384-415. 
[6] M. Ikawa, Decay of solutions of the wave equation in the exterior of two convex obstacles, Osaka J. Math. 19 (1982), no. 3, 459-509.

[7] - Decay of solutions of the wave equation in the exterior of several convex bodies, Ann. Inst. Fourier (Grenoble) 38 (1988), no. 2, 113-146.

[8] P. D. Lax, C. S. Morawetz, and R. S. Phillips, The exponential decay of solutions of the wave equation in the exterior of a star-shaped obstacle, Bull. Amer. Math. Soc. 68 (1962) 593-595.

[9] G. Lebeau, Équation des ondes amorties, in Algebraic and geometric methods in mathematical physics (Kaciveli, 1993), Vol. 19 of Math. Phys. Stud., 73-109, Kluwer Acad. Publ., Dordrecht (1996).

[10] C. S. Morawetz, The decay of solutions of the exterior initial-boundary value problem for the wave equation, Comm. Pure Appl. Math. 14 (1961) 561-568.

[11] C. S. Morawetz, J. V. Ralston, and W. A. Strauss, Decay of solutions of the wave equation outside nontrapping obstacles, Comm. Pure Appl. Math. 30 (1977), no. 4, 447-508.

[12] S. Nonnenmacher and M. Zworski, Quantum decay rates in chaotic scattering, to appear, Acta Math. (2007)

[13] - Semiclassical resolvent estimates in chaotic scattering, preprint (2009),

[14] J. V. Ralston, Trapped rays in spherically symmetric media and poles of the scattering matrix, Comm. Pure Appl. Math. 24 (1971) 571-582.

[15] B. R. Vaŭnberg, Asymptotic methods in equations of mathematical physics, Gordon \& Breach Science Publishers, New York (1989), ISBN 2-88124-664-8. Translated from the Russian by E. Primrose.

[16] G. Vodev, Local energy decay of solutions to the wave equation for nontrapping metrics, Ark. Mat. 42 (2004), no. 2, 379-397.

Department of Mathematics, Massachusetts Institute of Technology, 77 Mass. Ave. CAmbridge, MA 02130, USA

E-mail address: hans@math.mit.edu 